\title{
Perceptual invariance and the psychophysical law
}

\author{
HOSEYIN YILMAZ \\ ARTHUR D. LITTLE, INC., CAMBRIDGE, MASSACHUSETTS
}

The psychophysical power law, demonstrated empirically by Stevens, is derivable from two postulates concerning (1) the tendency of perceptual organizations to model the environment and (2) the tendency for perceptual relations to remain constant. The two postulates appear to rule out logarithmic laws of the Fechner type. The theory is extendable to multidimensional perceptions such as those encountered in speech and color vision. The power transformations produced by the sense organs may be of such form that they produce a perceptual space in which subsequent transformations need be only linear.

The rivalry between the power law of Stevens (1957) and the logarithmic law of Fechner (1860) lingers on despite the experimental evidence that sensed or perceived magnitude increases as a power function of stimulus magnitude (Stevens \& Galanter, 1957; Ekman \& Sjöberg, 1965). The point at issue has a bearing both on theoretical conceptions and on such practical matters as the design of equipment. For example, in the design of a vowel recognition machine, there arose the problem of devising a power law amplifier and a mel scale frequency filter in order to imitate the transformations of the human auditory system (Yilmaz, 1966).

Although the power law has gained ground, there remains considerable resistance, probably for several reasons. One is the inertia created by a century of allegiance to Fechner's logarithmic law, which filled a theoretical need and therefore became the foundation of psychophysical thinking. Indeed, Fechner's law appeared to follow so naturally from Weber's law that there grew up the semblance of a deductive theory. In a science that strives to become like physics, the appeal of a deductive approach should not be underestimated. Indeed, a similar approach motivates the present inquiry.

The empirical generality of Stevens' power law, as evidenced by the fact that it seems to hold in many if not all sense modalities (Stevens, 1961a), poses a challenge, inviting us to explore those basic principles in terms of which it may be better understood. The present purpose is to formulate a theoretical framework in which the power law finds a straightforward derivation. The framework is suggested by the requirements of evolutionary adaptation -in particular, the notion that perceptual systems encode the environment in maximally useful ways.

\section{Derivation of the Power Law}

The derivation is based on two postulates which were originally part of a larger set designed to account for the perceptual organization of speech sounds (Yllmaz, 1966). The larger set had its roots in an evolutionary approach to perception theory, an approach that had been previously applied to a derivation of the structure of color vision (Yilmaz, 1962). In the course of the construction of the speech theory, it became evident that two of the stated postulates automatically implied a power law. That was a pleasant surprise, because in a related but slightly different setting, the author had regarded the logarithmic law as an admissible alternative to the power law (Yilmaz, 1964).

The two postulates needed for the present purpose are: (1) Perceptual organizations tend to model the environment in a homological manner; (2) Percepts tend to remain invariant under environmental variations.

The use of the phrase "tend to" is meant to suggest that living organisms are noisy devices and that strictly veridical percepts may be only approximated. The two postulates express rather general functional principles whose implications need to be spelled out in more explicit form.

The first postulate implies that, to a physical stimulus variable $i$, there corresponds a sensory variable $s$. In other words, variable $i$ is imaged or represented by the variable $s$. The second postulate implies that sensory variables exhibit a relativity property such that, when the values of a physical variable undergo a systematic change, the values of the sensory variable change in such a way as to leave the perceptual content of a situation invariant. This principle attributes perceptual significance to the relations among stimuli $\left(i_{1}, i_{2}\right),\left(i_{2}, i_{3}\right), \ldots$, as well as to the magnitudes of the stimuli.

Many variables undergo systematic changes in typical perceiving situations. For example, light intensity may vary from high to low, thereby affecting all the intensities reflected from objects in the visual field by a constant positive factor $K$. Similarly, the intensity of speech may be high or low, depending on the speaker or the distance of the speaker. Again, the temperature of the room, the color of the illumination, the spectrum of an ambient noise, and many other factors may each add systematic variations to the physical variables to which the organism is trying to respond.

Let us consider, then, a relational physical property $f\left(i_{1}, i_{2}\right)$ that the organism finds it useful and survival-promoting to model in its perceptual response. The internal relational property $P\left(s_{1}, s_{2}\right)$, which may be called a percept, will be a representation of the external relational property. Omitting a scale parameter that depends only on units, we may write

$$
P\left(s_{1}, s_{2}\right)=f\left(i_{1}, i_{2}\right)
$$

We next consider the second of the two postulates. It states in effect that environmental stimulus changes tend to make no difference to relational percepts. Accordingly, in the aforementioned type of multiplicative variation, $P\left(s_{1}, s_{2}\right)$ will be invariant under the substitution $i \rightarrow K i$, that is,

$$
P\left(s_{1}, s_{2}\right)=f\left(i_{1}, i_{2}\right)=f\left(K i_{1}, K i_{2}\right)
$$

The desired invariance will be guaranteed if, as one possibility, $f$ is any function of the ratio $i_{2} / i_{1}$ 


$$
f\left(i_{1}, i_{2}\right)=f\left(i_{2} / i_{1}\right)=f\left(i_{1}-1 \cdot i_{2}\right)
$$

Finally, in accordance with the first postulate, we note that $P\left(s_{1}, s_{2}\right)$ is essentially a homological model, or analogue, of the external relational property $f\left(i_{1}, i_{2}\right)$. Therefore, in order to maintain close correspondence between the external world and the internal model, the same invariance property under magnitude substitution $s \rightarrow \lambda s$ will tend to be realized. In other words, $P$ is a function of the ratio $s_{2} / s_{1}$ of the internal or perceptual variables. From Eq. 1 we then have

$$
P\left(s_{2} / s_{1}\right)=f\left(i_{2} / i_{1}\right)
$$

Let $i_{1}=i, i_{2}=i+\Delta i$, and similarly $s_{1}=s, s_{2}=s+\Delta s$, and write Eq. 4 as

$$
P\left(1+\frac{\Delta s}{s}\right)=f\left(1+\frac{\Delta i}{i}\right)
$$

with boundary condition $P(1)=f(1)$. Expanding by Taylor's series and keeping only the first-order terms (which is appropriate if $\Delta i$ and $\Delta s$ are small compared to $i$ and $s$ ),

$$
P(1)+P^{\prime}(1) \frac{d s}{s}=f(1)+f^{\prime}(1) \frac{d i}{i}
$$

where $P^{\prime}(1)$ and $f^{\prime}(1)$ are derivatives evaluated at $s_{2}=s_{1}$ and $i_{2}=i_{1}$ and therefore constants. By using the boundary condition and integrating, we obtain

$$
s=A i^{\alpha}, \quad \alpha=f^{\prime}(1) / P^{\prime}(1)
$$

which is the power law of Stevens.

Note that the derivation of this first-order law makes no use of the concept of the just noticeable difference (jnd) or resolving power. Nor is Weber's law required. The $\Delta i$ in Eq. 5 differs from a jnd in the same sense that a differential differs from a measure of dispersion. In other words, $\Delta i$ is a difference, whereas a jnd is a measure of variability, scatter, or noise.

What would happen to the psychophysical law if the relaticnal process concerned differences rather than ratios? Under the postulate of homological modeling, $P\left(s_{1}, s_{2}\right)$ cannot satisfy an additive invariance unless $f\left(i_{1}, i_{2}\right)$ also satisfies such an invariance. An additive invariance leads, by a parallel series of arguments, not to Eq. 4 but to the formula

$$
P\left(s_{2}-s_{1}\right)=f\left(i_{2}-i_{1}\right)
$$

Then by a similar procedure of integration, $i_{1}=i, i_{2}=i+\Delta i, s_{1}=s, s_{2}=s+\Delta s$, etc., we arrive at a linear law

$$
s=A i+B, \quad A=f^{\prime}(0) / P^{\prime}(0)
$$

It is interesting that this rather different perceptual invariance results in a power law of unit exponent. Unit exponents are found in the perception of such continua as length, repetition rate, time durations, and the like.

It should, perhaps, be noted that the particular mathematical procedure used here to derlve the power law is not the only one possible. Alternative derivations may be framed, for example, in the language of functional equations, or of group theory. It was hoped that the use of Taylor's series would prove more familiar to the reader.

The foregoing development has examined two kinds of relational structures that may obtain in perception -the one based on ratios, and the other on differences. In neither case did the derivation lead to Fechner's logarithmic law. We may ask, therefore, under what circumstances a logarithmic law would be possible. As spelled out earlier (Yilmaz, 1964), if additive responses in perception are assumed to correspond to multiplicative changes in the stimulus, a derivation similar to that presented above will lead to a logarithmic law. The difficulty, of course, is that, by relating perceptual differences to stimulus ratios, we have violated the demands of the first of our two main postulates. The requirement that perceptual structures must model physical configurations may be better fulfilled if ratios among stimuli are made to correspond to ratios among percepts.

The physicist Plateau (1872) was probably the first to sense the unreasonableness of a requirement that perceptual differences should correspond to physical ratios. Without advancing a formal theory, Plateau conjectured a power law, a conjecture that he withdrew when he was later confronted with the data obtained by his friend Delboeuf. Delboeuf used a procedure (equisection) that has since been found to give results that depart systematically from the psychophysical power law (Stevens, 1961b).

\section{Concerning Weber's Law}

The theory set forth above seems to rule out Fechner's law, but it says nothing about Weber's Jaw-the law that states that the jnd is proportional to the stimulus magnitude. This may appear paradoxical, because Fechner's law was originally deduced from Weber's law. The questionable word here, however, is the word "deduced." As argued by many critics, the so called deduction was based on the unjustifiable assumption that a jnd may be considered mathematically as an exact differential. With this assumption set aside, Weber's law and Stevens' law can both be independently valid, and yet Fechner's law can be invalid. In order to clarify this point, an explicit example has been constructed to illustrate that Stevens' law does not impose any condition on the distribution of the jnd, and therefore does not say anything about the validity of Weber's law.

Let us consider the pitch-frequency relation as given below by the power function of Eq. (10). This relation states that to every value of $v$ there corresponds a value $p$, and that in order to double the pitch sensation we must more than double $\left(v-v_{0}\right)$. Let us assume that frequency detection is performed in the ear by a series of resonators, and that the width of the resonance curve of each resonator corresponds to a jnd. The jnd becomes the resolving power or resolution error of the resonator in question. Now, without changing the number or the order of the resonators, we could change the width of the resonance curve of each resonator by introducing different degrees of damping. Each resonator would still respond to its appropriate frequency. In other words, we could keep the power law relation unaltered, but change the jnd to fit Weber's law or any other relation desired. This is not to say that Weber's law is not valid. But if it is valid, the reason 
for its validity is not to be found in the form of the psychophysical law, because the two laws are independent of each other. Actually, to a fair approximation, Weber's law holds in the upper but not in the lower part of the frequency range (Stevens \& Davis, 1938).

One might object that pitch is not a prothetic continuum, or that the jnd does not represent a resonance-type resolving power. However, if we consider loudness instead of pitch, and noise power instead of frequency resolution, the argument still applies. The law governing the jnd concerns variability or uncertainty, and has nothing to do with the form of the psychophysical function. To the extent that Weber's law is valid, it emerges as an independent experimental finding: It stands or falls with experiments concerning its own domain of phenomena -the statistical determination of the degree to which two stimuli can be told apart. As a corollary to the foregoing argument, it seems clear that the derivations of the power law based on the premises of Brentano or Guilford are not acceptable, because they start, one way or another, with an assumption about the jnd (Brentano, 1874; Guilford, 1932).

The clear separation of the jnd and Weber's Iaw from the psychophysical power function leaves us with the problem of why Weber's law holds, or is at least approximated, in so many perceptual systems.

Perhaps the answer is to be found in the rather widespread physical principle that relative error tends to be constant. Absolute error is constant only under special circumstances. Accuracies of measurement are generally stated not in terms of so many units, but in terms of one part in so many units. This relativity of error is easy to understand. We can measure to a micron when small distances are involved, but not when it is a question of the distance from New York to Moscow.

We expect that the size of the error, variability, or noise will increase with the physical magnitude that is being measured. The same rule applies, at least roughly, when living organisms are involved. One of the classical methods for measuring the jnd is the method of average error. A person is asked to adjust one stimulus to make it appear equal to another stimulus. He makes many settings, and the average deviation from the mean of the settings is taken as the jnd. When the match is to a small stimulus, the average error is smaller than when the match is to a large stimulus. In its general form, Weber's law says simply that the deviation from the mean will increase linearly with the stimulus magnitude. This is a principle that is approximated in much of physics.

Granted then that Weber's law is independent of the form of the psychophysical power function, let us return briefly to the problem of pitch, which is said to be a metathetic (qualitative) rather than a prothetic (quantitative) continuum. The pitch continuum seems to satisfy the relativity implication of our second postulate, since we know that speech perception is invariant under pitch variations. Thus a man, a woman, or a child may speak with different pitches, and yet the speech is understood, or a melody may remain invariant under a change of key. Indeed, for the speech range $(300-4000 \mathrm{~Hz})$, the mel scale of pitch sensation as determined experimentally by Stevens and Volkmann (1940) can be approximated by a power function.

$$
p=k\left(v-v_{0}\right)^{0.7}
$$

Such a function may seem to raise the question whether pitch is a prothetic variable. It should be noted, however, that the fact of being a power function was not one of the criteria used by Stevens in 1957 to define a prothetic continuum. He later stated explicitly that on a metathetic continuum a power function may or may not be found (Stevens, 1958). Hence there seems to be no reason why pitch, at least in the speech range, should not exhibit the invariance properties that entail a power function. Furthermore, pitch and loudness take part in a multidimensional organization that may be thought of as speech space, a space in which there are relations and overall relativity requirements. In other words, because of their relations and transformations within a multidimensional realm, somevariables, such as pitch, may behave the way prothetic variables do.

\section{Mulfidimensional Psychophysics}

The derivation carried out above involves only one modality, and the perceptual variables $s_{1}$ and $\mathrm{s}_{2}$ represent different values of the same variable. When two continua are involved, as in cross-modality comparisons, $s_{1}$ and $s_{2}$ may represent entirely different modes, say, $s_{1}$ may equal brightness, and $s_{2}$, loudness.

The observer in the cross-modality experiment may, as an example, adjust the intensity of a sound to make its loudness seem as strong as the brightness of a given light. Or he may vary the light to match the sound (Stevens \& Marks, 1965). By means of systematic cross-modality comparisons a transitive network of power functions may be mapped out, such that the exponent of the matching function is equal to the ratio of the exponents of the two sense modalities that are being compared (Stevens, 1966). The cross-modality formula of Stevens may be written

$$
\frac{\log i_{2}}{\log i_{1}}=\frac{\alpha_{2}}{\alpha_{1}}
$$

where $\alpha_{1}$ and $\alpha_{2}$ are the exponents of the sensory continua for which $i_{1}$ and $i_{2}$ are the physical stimuli.

The intermediate situation, where $s_{1}$ and $s_{2}$ may be different perceptual continua belonging to the same sense modality, seems to require a separate discussion. To clarify the issue here, let us consider the farniliar color domain where hue, brightness, and saturation are present simultaneously. These three variables may be regarded as different continua, but they are nevertheless closely related. All three may emerge from the same light distribution, and perceptually they form a closely knit but multidimensional structure. An attempt will be made to show that the 
postulates and theory proposed for a single dimension are applicable also to the multidimensional case.

For the sake of simplicity, let us consider the cartesian coordinates $c$, $a, b$ of a color sample. The coordinates may be regarded as an orthogonal system. Let boldface $i_{1}$ and $i_{2}$ be vectors (or matrices) representing the three coordinates of the color sample. In the general case, the transformation of the illuminant may involve more than a simple scale change of intensity. For example, if a cloud covers the sun, not only does the intensity of light change, but the spectrum of the illumination also changes from yellowish sunlight to blue-sky quality. Let $L$ be the transformation in question. We may assume this to be a similarity transformation, which can always be written as a product $L=k U$ of a number $k$ and an orthogonal matrix $U$. Thus

$$
L=k U, \quad U U^{+}=1
$$

where $k$ is the scale change and $U$ is an orthogonal matrix having to do with the chromatic part of the transformation. $U^{+}$is the transpose of the matrix $U$. (Note: An orthogonal transformation preserves length and implies that the underlying space is metric. Examples are rotation of axes on a plane, which preserves $x^{2}+y^{2}$, and Lorentz transformations, which preserve $c^{2} t^{2}-x^{2}$.)

The following generalization runs parallel to and lies entirely within the philosophy of the previous considerations.

$$
\begin{gathered}
P\left(\mathrm{~s}_{1}, \mathrm{~s}_{2}\right)=f\left(\mathbf{i}_{1}, \mathrm{i}_{2}\right) \\
P\left(\mathrm{~s}_{1}, \mathrm{~s}_{2}\right)=f\left(L \mathbf{i}_{1}, L \mathbf{i}_{2}\right)=f\left[\left(L \mathbf{i}_{1}\right)^{-1} \cdot L \mathbf{i}_{2}\right]
\end{gathered}
$$

Then, as in the earlier development, the second of the two basic postulates requires an internal transformation $N=\mu W$ for the sensory quantity $\mathrm{s}$. Thus, we can define a new unitary or orthogonal transformation $W$,

$$
N=\mu W, \quad W W^{+}=1
$$

so that, without going through the intermediate steps, we may infer the generalized form of Eq. 4 as

$$
P\left[\left(W s_{1}\right)^{-1} \cdot W_{s_{2}}\right]=f\left[\left(U \mathrm{i}_{1}\right)^{-1} \cdot U_{\mathbf{i}_{2}}\right]
$$

For vectors and matrices, such expressions as $s_{1}^{-1}$ or $i_{1}{ }^{-1}$ have to be reinterpreted. Let us define the reciprocals

$$
\mathrm{s}=|s| \sigma, \quad \sigma^{-1}=\frac{\sigma^{+}}{|s|}, \quad \mathrm{i}=|i| \epsilon, \quad \mathrm{i}^{-1}=\frac{\epsilon^{+}}{|i|}
$$

where $|s|$ and $|i|$ are absolute magnitudes. In the sense of tensor calculus, $|s| \sigma^{+}$and $|i| \epsilon^{+}$may be interpreted as covariant descriptions if $s$ and $i$ are considered contravariant vectors. Substituting into Eq. 16 and using the orthogonality of both $U$ and $W$ we find

$$
P\left(\frac{\left|s_{2}\right|}{\left|s_{1}\right|} \sigma_{1}+\cdot \sigma_{2}\right)=f\left(\frac{\left|i_{2}\right|}{\left|i_{1}\right|} e_{1}+\cdot \epsilon_{2}\right)
$$

Except for the scalar products $\sigma_{1}^{+} \cdot \sigma_{1}$ and $\epsilon_{1}{ }^{+} \cdot e_{2}$, this formula is the the same as Eq. 4. However, the scalar product is nothing but a cosine, since the vectors are unit vectors. To a first-order approximation, a cosine function is unity, since $\cos \theta=1-\frac{\theta^{2}}{2 !}+\frac{\theta^{4}}{4 !} \ldots$. Therefore, by a procedure of integration similar to that used previously, we obtain, to a first-order approximation, the power law

$$
|s|=A|i| \alpha
$$

On the other hand, by looking at Eq. 16 we may infer that the orthogonal transformation $W$ also satisfies a power law

$$
W=U^{\beta}
$$

where $\beta$ is not necessarily equal to $\alpha$. The conclusion seems to be that, in the multidimensional situation, the vectors obey, to a first-order approximation, a more general and composite type of Stevens law, and reduce to Eq. 7 in the special case of a single scalar variable.
Although the foregoing development was intended to show that the power law is generalizable to multidimensional situations, the resulting equations (19) and (20) may also prove useful in other ways, in particular for the construction of a multidimensional psychophysics applicable to such domains as color and speech. Thus, in order to obtain the author's color theory (1962), we need only to make the following identifications: (1) color space is threedimensional, and (2) brightness is positive definite (there are no negative brightnesses). It would follow that the structure of the transformation is Lorentzlike. By this procedure we find that a theory that was previously constructed in a laborious fashion from several physical and evolutionary (adaptive) postulates can be fitted directly into the framework provided by Eqs. (19) and (20).

The same equations may also provide a framework for the log-log coordinate system used by Land $(1959,1964)$ to describe the color renditions obtained with two-color projections. The relative intensities of the two stimuli (short wave and long wave) provide the two logarithmic coordinates. In addition to being power functions, straight lines in this coordinate system have special perceptual significance. For example, the achromatic colors, as seen in the projected image, tend to fall on the median diagonal. The hues fall on either side of the diagonal, and each hue tends to fall on a different line. However, if the two colors are projected through identical transparencies, the viewer observes a wash having only the color of the two combined illuminants. These so called "achromatic phenomena" of Land seem to be related to Eqs. (19) and (20), because the equations imply a power law for each color vector in the color space. Some of Land's recent work has explored color invariance under two kinds of transformations: the wavelengths used in the two-color projections and the relative intensities of the two projected images.

It should be pointed out that under the isolated conditions of a color seen in a dark field, which is quite different from the two-color projection described above, the power law seems also to apply. The apparent saturation of a color is a power function of the physical purity of the stimulus. Similarly, hue (e.g., redness of a red-yellow mixture) is a power function of the mixture ratio of the two adjacent primary hues (Indow \& Stevens, 1966).

\section{Infernal Linearity}

The organism tries to encode its perceptual information in a form that will be most useful. The information should depict the environment even when the environmental variables undergo large changes involving complex interactions. What is required is a high degree of perceptual Invariance inside the organism despite continual changes in the outside 
world-a world that the organsim senses only through its end-organ transducers. The input-output characteristics of some transducers appear to be quite nonlinear. In some of the sense modalities the nonlinearity may serve the useful purpose of compressing enormous stimulus ranges, such as those encountered in vision and hearing, in such a way that the system beyond the transducer may operate essentially linearly. The invariance of percepts can be more easily maintained if the required transformations in perceptual space remain linear. For this reason, it may prove advantageous to regard the sensory power law transformation as nature's way of creating linear simplicity in the inner world in the face of complex nonlinearities in the outer world.

As a corollary of this principle, we should expect that quantitative relations among percepts may sometimes prove to be simpler when they are expressed in terms of subjective variables than when they are expressed in terms of stimulus variables. Two examples may be cited. Studies in Stockholm have shown that apparent similarity may be expressed as a simple mathematical function of the percepts being compared. The simplicity was lost when the physical stimulus values were employed. As Ekman wrote, "These results seem to indicate the possibility of discovering psychological mechanisms which may turn out to be rather simple when they are studied on the proper-that is to say, subjectivelevel." (Ekman, 1961).

As a second example, in studies of auditory perception it was found that the creation of subjective scales for the apparent loudness, apparent volume, and apparent density of sounds leads directly to a simple relation: Apparent loudness is proportional to apparent volume times apparent density (Stevens, Guirao, \& Slawson, 1965). When expressed in terms of the stimulus dimensions, frequency, and intensity, the relation among the three subjective attributes becomes obscure and the simple linear relation disappears.

Does the foregoing thesis imply that, beyond the peripheral sense organs, the nervous system performs only linear operations? Neurophysiology has not as yet obtained a definite answer to that question, but interesting affirmative evidence has been cited by Sir John Eccles in a recent Ciba Symposium (de Reuck \& Knight, 1966). After describing the convergence of the synapses on pyramidal cells, he concluded that "there is no great impediment to the idea that when all these factors are added up, the transfer functions across the synaptic mechanism are approximately linear." Professor V. B. Mountcastle added, "The interesting point for me here is the great importance that we must now place upon the transducer process itself, at the periphery. That is, the whole functional relationship of the response to the intensity of a stimulus is set at the first stage, which I believe is a new idea...."

Thus it may turn out that the complexion of the problem posed by the neurophysiologists may find itself altered to accord more directly with the notion of simple linear invariances in the central nervous system. The creation of the linear invariances may be the job of the sense organs with their power law operating characteristics.

\section{References}

Bishop, P. O. Central nervous system: Afferent mechanisms and perception. In Annual Review of Physiology. Vol. 29. Palo Alto, California: Annual Reviews, 1967.

Brentano, F. Psychologie vom empirischen Standpunkte, Bd, 1. Leip zig: Duncker \& Humblot, 1874.

de Reuck, A. V. S., \& Knight, J. (Eds.) Touch, heat and pain, A Ciba Foundation Symposizm. London: Churchill, 1966. See discussion by Sir John Eccles and V. B. Mountcastle, p. 23.

Ekman. G. Some aspects of psychophysical research. In W. A. Rosenblith (Ed.), Sensory communication. Cambridge: M.I.T. Press, 1961. P. 46.

Ekman, G., \& Sjoberg, L. Scaling. Ann. Rev. Psychol., 1965, 16, $451-474$.

Fechner, G.T. Elemente der Psychophysik. Leipzig: Breitkopf \& Härtel, 1860. English trans, by H. E. Adler, edited by D. H. Howes and E. G. Boring, New York: Holt, Rinehart and Winston, 1966.

Guilford, J. P. A generalized psychophysical law. Psychol. Rev., $1932,39,73-85$.

Indow, T., \& Stevens, $\mathbf{S}$. S. Scaling of saturation and hue. Percept. \& Psychophys., 1966, 1, 253-272.

Land, E. H. Color vision and the natural image. Part I. Proc. Nat. Acad. Sci., 1959, 45, 115-129.

Land, E. H. The retinex. Amer. Sci., 1964, 52, 247-264.

Plateau, J. Sur la mesure des sensations physiques, et sur la loi qui lie l'intensité de ces sensations a l'intensité de la cause excitante. Bull. Acad. Roy. Belg. 1872, 33 (ser. 2), 376-388.

Stevens, J. C., \& Marks, L. E. Cross-modality matching of brightness and loudness. Proc. Nat. Acad. Sci., 1965, 54, 407-411.

Stevens, S. S. On the psychophysical law. Psychol. Rev., 1957, 64, 153-181.

Stevens, S. S. Problems and methods of psychophysics. Psychol. Bull., 1958, 55, 177-196.

Stevens, S. S. The psychophysics of sensory function. In W. A. Rosenblith (Ed.), Sensory communication. Cambridge: M.I.T. Press, 1961a.

Stevens, S. S. To honor Fechner and repeal his Iaw. Science, $1961 \mathrm{~b}, 133,80-86$.

Stevens, S. S. Matching functions between loudness and ten other continua. Percept. \& Psychophys., 1966, 1, 5-8.

Stevens, S. S., \& Davis, H. Hearing: Its psychology and physiology. New York: John Wiley \& Sons, 1938.

Stevens, S. S., \& Galanter, E. H. Ratio scales and category scales for a dozen perceptual continua. J. exp. Psychol., 1957, 54, 377411.

Stevens, S. S., Guirao, M., \& Slawson, A. W. Loudness, a product of volume times density. J. exp. Psychol., 1965, 69, 503-510. Stevens, S. S., \& Volkmann, J. The relation of pitch to frequency: a revised scale. Amer. J. Psychol., 1940, 53, 329-353.

Yilmaz, H. On color vision and a new approach to general perception. In E. E. Bernard \& M. R. Kare (Eds.), Biological prototypes and synthetic systems. Vol. I. New York: Plenum Press, 1962. Pp. 126-141. 
Yilmaz, H. On the laws of psychophysics. Bull. Math. Biophys., $1964,26,235-227$.

Yilmaz, h. A program of research directed toward the efficient and accurate machine recognition of human speech. Report C-68366. Cambridge: Arthur D. Little, Inc., December 14, 1966.

\section{Note}

1. Work supported by NASA, Electronic Research Center, Cambridge, Massachusetts, under Contract NAS-129. Part of the paper was written at the Center for Theoretical Studies, University of Miami, Coral Gables, Florida. I would like to thank Behram Kursunoglu and the University of Miami for their hospitality at the Center for Theoretical Studies, and the officials of Arthur D. Little, Inc., for making my visit to the Center possible. Gratitude is also due S. S. Stevens and A. G. Emslie for illuminating discussions and many essential suggestions.

(Accepted for publication July 25, 1967.) 